\title{
Decoherence of Flux Qubits Coupled to Electronic Circuits
}

\author{
F.K. Wilhelm ${ }^{1}$, M.J. Storcz ${ }^{1}$, C.H. van der $\mathrm{Wal}^{2}$, C.J.P.M. Harmans ${ }^{3}$, and \\ J.E. Mooij ${ }^{3}$ \\ 1 Sektion Physik and CeNS, Ludwig-Maximilians-Universität, 80333 München, \\ Germany \\ 2 Dpt. of Physics, Harvard University, Cambridge, MA 02138, USA \\ 3 Dpt. of Nanoscience, Delft University of Technology, 2600 GA Delft, \\ Netherlands
}

\begin{abstract}
On the way to solid-state quantum computing, overcoming decoherence is the central issue. In this contribution, we discuss the modeling of decoherence of a superonducting flux qubit coupled to dissipative electronic circuitry. We discuss its impact on single qubit decoherence rates and on the performance of two-qubit gates. These results can be used for designing decoherence-optimal setups.
\end{abstract}

Quantum computation is one of the central interdisciplinary research themes in present-day physics [1]. It promises a detailed understanding of the often counterintuitive predictions of basic quantum mechanics as well as a qualitative speedup of certain hard computational problems. A generic, although not necessarily exclusive, set of criteria for building quantum computers has been put forward by DiVincenzo [2]. The experimental realization of quantum bits has been pioneered in atomic physics, optics and NMR. There, the approach is taken to use microscopic degrees of freedom which are well isolated and can be kept quantum coherent over long times. Efficient controls are attached to these degrees of freedom. Even though these approaches are immensely succesful demonstrating elementary operations, it is not evident how they can be scaled up to macroscopic computers.

Solid-state systems on the other hand have proven to be scalable in present-day classical computers. Several proposals for solid-state based quantum computers have been put forward, many of them in the context of superconductors [3]. As solid-state systems contain a macroscopic number of degrees of freedom, they are very sensitive to decoherence. Mastering and optimizing this decoherence is a formidable task and requires deep understanding of the physical system under investigation. Recent experimental success $[4,5]$ suggests that this task can in principle be performed.

In this contribution, we are going to study decoherence of superconducting qubits coupled to an electromagnetic environment which produces JohnsonNyquist noise. We show, how the decoherence properties can be engineered by carefully designing the environmental impedance. We will discuss how the decoherence affects the performance of a CNOT operation. 


\section{Superconducting Flux Qubits}

Superconducting qubits [3-6] are very well suited for the task of solid-state quantum computation, because two of the most obvious decoherence sources in solid-state systems are supressed: Quasiparticle excitations experience an energy gap and phonons are frozen out at low temperatures [7]. The computational Hilbert space is engineered using Josephson tunnel junctions that are characterized by two competing energy scales: The Josephson coupling of a junction with critical current $I_{\mathrm{c}}, E_{\mathrm{J}}=I_{\mathrm{c}} \Phi_{0} / 2 \pi$, and the charging energy $E_{\mathrm{ch}}=2 e^{2} / C_{\mathrm{J}}$ of a single Cooper pair on the geometric capacitance $C_{\mathrm{J}}$ of the junction. Here $\Phi_{0}=h / 2 e$ is the superconducting flux quantum. There is a variety of qubit proposals classified by the ratio of this energies. Whereas another contribution in this volume [8] focuses on the case of charge qubits, $E_{\mathrm{ch}}>E_{\mathrm{J}}$, this contribution is motivated by flux qubit physics, $E_{\mathrm{J}}>E_{\mathrm{ch}}$. However, most of the discussion has its counterpart in other superconducting setups as well. Specifically, we discuss a three junction

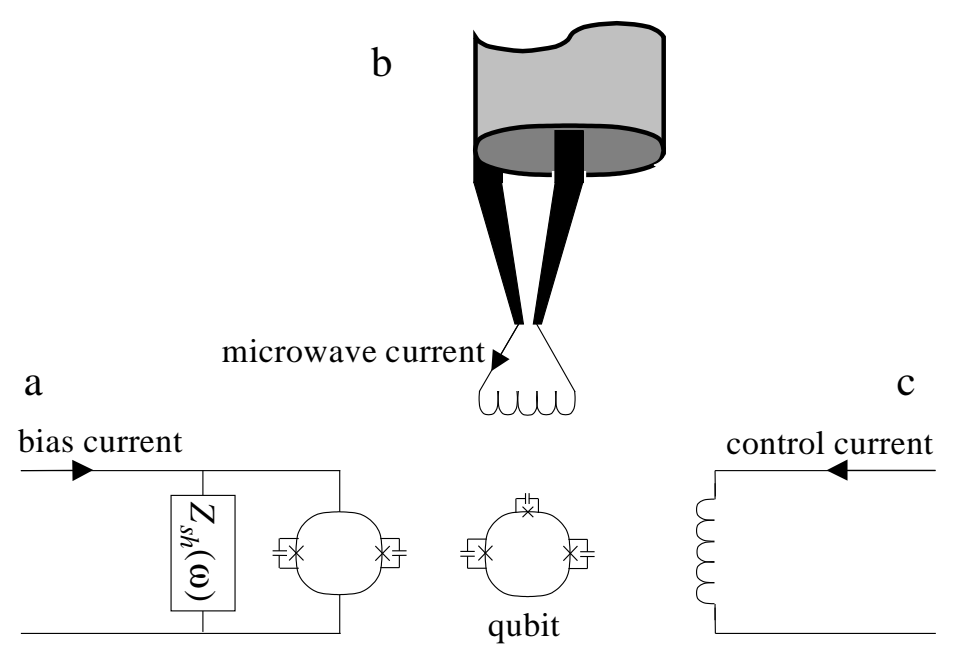

Fig. 1. Experimental setup for measurements on a flux qubit. The qubit (center) is a superconducting loop that contains three Josephson junctions. It is inductively coupled to a DC-SQUID (a), and superconducting control lines for applying magnetic fields at microwave frequencies (b) and static magnetic fields (c). The DC-SQUID is realized with an on-chip shunt circuit with impedance $Z(\omega)$. The circuits a)-c) are connected to filtering and electronics (not drawn)

qubit $[6,9]$, a micrometer-sized low-inductance superconducting loop containing three Josephson tunnel junctions (Fig. 1). By applying an external flux $\Phi_{q}$ a persistent supercurrent can be induced in the loop. For values where $\Phi_{q}$ 
is close to a half-integer number of flux quanta, two states with persistent currents of opposite sign are nearly degenerate but separated by an energy barrier. We will assume here that the system is operated near $\Phi_{q}=\frac{1}{2} \Phi_{0}$. The persistent currents in the classically stable states have here a magnitude $I_{\mathrm{p}}$. Tunneling through the barrier causes a coupling between the two states, and at low energies the loop can be described by a Hamiltonian of a two state system $[6,9]$,

$$
\hat{H}_{q}=\frac{\varepsilon}{2} \hat{\sigma}_{z}+\frac{\Delta}{2} \hat{\sigma}_{x}
$$

where $\hat{\sigma}_{z}$ and $\hat{\sigma}_{x}$ are Pauli matrices. The two eigenvectors of $\hat{\sigma}_{z}$ correspond to states that have a left or a right circulating current and will be denoted as $|L\rangle$ and $|R\rangle$. The energy bias $\varepsilon=2 I_{p}\left(\Phi_{q}-\frac{1}{2} \Phi_{0}\right)$ is controlled by the externally applied field $\Phi_{q}$. We follow [10] and define $\Delta$ as the tunnel splitting at $\Phi_{q}=$ $\frac{1}{2} \Phi_{0}$, such that $\Delta=2 W$ with $W$ the tunnel coupling between the persistentcurrent states. This system has two energy eigen values $\pm \frac{1}{2} \sqrt{\Delta^{2}+\varepsilon^{2}}$, such that the level separation $\nu$ gives $\nu=\sqrt{\Delta^{2}+\varepsilon^{2}}$. In general $\Delta$ is a function of $\varepsilon$. However, it varies on the scale of the single junction plasma frequency, which is much above the typical energy range at which the qubit is operated, such that we can assume $\Delta$ to be constant for the purpose of this paper.

In the experiments $\Phi_{q}$ can be controlled by applying a magnetic field with a superconducting coil at a distance from the qubit and for local control one can apply currents to superconducting lines, fabricated on-chip in the vicinity of the qubit. The qubit's quantum dynamics will be controlled with resonant microwave pulses (i. e. by Rabi oscillations). In recent experiments the qubits were operated at $\varepsilon \approx 5 \Delta$ or $\varepsilon \approx 0[4,9]$. The numerical values given in this paper will concentrate on the former case. At this point, there is a good trade-off between a system with significant tunneling, and a system with $\hat{\sigma}_{z}$-like eigenstates that can be used for qubit-qubit couplings and measuring qubit states [6]. The qubit has a magnetic dipole moment as a result of the clockwise or counter-clockwise persistent current The corresponding flux in the loop is much smaller than the applied flux $\Phi_{q}$, but large enough to be detected with a SQUID. This will be used for measuring the qubit states. For our two-level system Eq. (1), this means that both manipulation and readout couple to $\hat{\sigma}_{z}$. Consequently, the Nyquist noise produced by the necessary external circuitry will couple in as flux noise and hence couple to $\hat{\sigma}_{z}$, giving $\epsilon$ a small, stochastically time-dependent part $\delta \epsilon(t)$.

Operation at $\varepsilon \approx 0$ has the advantage that the flux noise leads to less variation of $\nu$. In the first experiments [4] this has turned out to be crucial for observing time-resolved quantum dynamics. Here, the qubit states can be measured by incorporating the qubit inside the DC-SQUID loop. While not working that out in detail, the methods that we present can also be applied for the analysis of this approach. This also applies to the analysis of the impact of electric dipole moments, represented by $\hat{\sigma}_{x}$. With $E_{\mathrm{ch}} \ll E_{J}$, these couple much less to the circuitry and will hence not be discussed here. 
As the internal baths are well suppressed, the coupling to the electromagnetic environment (circuitry, radiation noise) becomes a dominant source of decoherence. This is a subtle issue: It is not possible to couple the circuitry arbitrarily weakly or seal the experimental setup, because it has to remain possible to control the system. One rather has to engineer the electromagnetic environment to combine good control with low unwanted back-action.

Any linear electromagnetic environment can be described by an effective

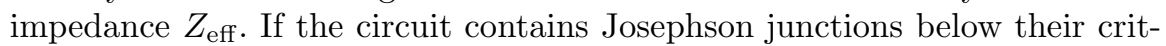
ical current, they can be included through their kinetic inductance $L_{\text {kin }}=$ $\Phi_{0} /\left(2 \pi I_{\mathrm{c}} \cos \bar{\phi}\right)$, where $\bar{\phi}$ is the average phase drop across the junction. The circuitry disturbs the qubit through its Johnson-Nyquist noise, which has Gaussian statistics and can thus be described by an effective Spin-Boson model [11]. In this model, the properties of the oscillator bath which forms the environment are characterized through a spectral function $J(\omega)$, which can be derived from the external impedance. Note, that other nonlinear elements such as tunnel junctions which can produce non-Gaussian shot noise are generically not covered by oscillator bath models.

As explained above, the flux noise from an external circuit leads to $\epsilon=$ $\epsilon_{0}+\delta \epsilon(t)$ in Eq. (1). We parametrize the noise $\delta \epsilon(t)$ by its power spectrum

$$
\langle\{\delta \epsilon(t), \delta \epsilon(0)\}\rangle_{\omega}=\hbar^{2} J(\omega) \operatorname{coth}\left(\hbar \omega / 2 k_{B} T\right) .
$$

Thus, from the noise properties calculated by other means one can find $J(\omega)$ as was explained in Detail in [12]. In this contribution, we would like to outline an alternative approach pioneered by Leggett [13], where $J(\omega)$ is derived from the classical friction induced by the environment. In reality, the combined system of SQUID and qubit will experience fluctuations arising from additional circuit elements at different temperatures, which can be treated in a rather straightforward manner.

\section{Decoherence from the Electromagnetic Environment}

\subsection{Characterizing the Environment from Classical Friction}

We study a DC-SQUID in an electrical circuit as shown in Fig. 1. It contains two Josephson junctions with phase drops denoted by $\gamma_{1 / 2}$. We start by looking at the average phase $\gamma_{\mathrm{ex}}=\left(\gamma_{1}+\gamma_{2}\right) / 2$ across the read-out SQUID. Analyzing the circuit with Kirchhoff rules, we find the equation of motion

$$
2 C_{J} \frac{\Phi_{0}}{2 \pi} \ddot{\gamma}_{\mathrm{ex}}=-2 I_{c, 0} \cos \left(\gamma_{i}\right) \sin \gamma_{\mathrm{ex}}+I_{\mathrm{bias}}-\frac{\Phi_{0}}{2 \pi} \int d t^{\prime} \dot{\gamma}_{\mathrm{ex}}\left(t^{\prime}\right) Y\left(t-t^{\prime}\right) .
$$

Here, $\gamma_{\text {in }}=\left(\gamma_{1}-\gamma_{2}\right) / 2$ is the dynamical variable describing the circulating current in the loop which is controlled by the flux, $I_{\text {bias }}$ is the bias current imposed by the source, $Y(\omega)=Z^{-1}(\omega)$ is the admittance in parallel to the whole SQUID and $Y(\tau)$ its Fourier transform. The SQUID is described by 
the junction critical currents $I_{\mathrm{c}, 0}$ which are assumed to be equal, and their capacitances $C_{\mathrm{J}}$. We now proceed by finding a static solution which sets the operation point $\gamma_{\text {in/ex }, 0}$ and small fluctuations around them, $\delta \gamma_{\text {in/ex }}$. The static solution reads $I_{\text {bias }}=I_{c, \text { eff }} \sin \gamma_{\mathrm{ex}, 0}$ where $I_{\mathrm{c}, \mathrm{eff}}=2 I_{c, 0} \cos \gamma_{i n, 0}$ is the effective critical current of the SQUID. Linearizing Eq. 3 around this solution and Fourier-transforming, we find that

$$
\delta \gamma_{\mathrm{ex}}(\omega)=\frac{2 \pi I_{\mathrm{b}} \tan \gamma_{\mathrm{in}, 0} Z_{\mathrm{eff}}(\omega)}{i \omega \Phi_{0}} \delta \gamma_{i}(\omega)
$$

where $Z_{\text {eff }}(\omega)=\left(Z(\omega)^{-1}+2 i \omega C_{\mathrm{J}}+\left(i \omega L_{\mathrm{kin}}\right)^{-1}\right)^{-1}$ is the effective impedance of the parallel circuit consisting of the $Z(\omega)$, the kinetic inductance of the SQUID and the capacitance of its junctions. Neglecting self-inductance of the SQUID and the (high-frequency) internal plasma mode, we can straightforwardly substitute $\gamma_{\text {in }}=\pi \Phi / \Phi_{0}$ and split it into $\gamma_{\text {in }, 0}=\pi \Phi_{\mathrm{x}, \mathrm{S}} / \Phi_{0}$ set by the externally applied flux $\Phi_{\mathrm{x}, \mathrm{S}}$ through the SQUID loop and $\delta \gamma_{\mathrm{i}}=\pi M_{\mathrm{SQ}} I_{\mathrm{Q}} / \Phi_{0}$ where $M_{\mathrm{SQ}}$ is the mutual inductance between qubit and the SQUID and $I_{\mathrm{Q}}(\varphi)$ is the circulating current in the qubit as a function of the junction phases, which assumes values $\pm I_{\mathrm{p}}$ in the classically stable states.

In order to analyze the backaction of the SQUID onto the qubit in the two-state approximation, Eq. (1), we have to get back to its full, continuous description, starting from the classical dynamcis. These are equivalent to a particle, whose coordinates are the two independent junction phases in the three-junction loop, in a two-dimensional potential

$$
\boldsymbol{C}\left(\Phi_{0} / 2 \pi\right)^{2} \ddot{\boldsymbol{\varphi}}=-\nabla U\left(\boldsymbol{\varphi}, \Phi_{x, q}+I_{\mathrm{S}} M_{\mathrm{SQ}}\right) .
$$

The details of this equation are explained in [6]. $C$ is the capacitance matrix describing the charging of the Josephson junctions in the loop, $U(\varphi)$ contains the Josephson energies of the junctions as a function of the junction phases and $I_{\mathrm{S}}$. is the ciculating current in the SQUID loop. The applied flux through the qubit $\Phi_{\mathrm{q}}$ is split into the flux from the external coil $\Phi_{x, q}$ and the contribution form the SQUID. Using the above relations we find

$$
I_{\mathrm{S}} M_{\mathrm{SQ}}=\delta \Phi_{\mathrm{cl}}-2 \pi^{2} M_{\mathrm{SQ}}^{2} I_{B}^{2} \tan ^{2} \gamma_{\mathrm{in}, 0} \frac{Z_{\mathrm{eff}}}{i \omega \Phi_{0}^{2}} I_{\mathrm{Q}}
$$

where $\delta \Phi_{\mathrm{cl}} \simeq M_{\mathrm{SQ}} I_{c, 0} \cos \gamma_{\mathrm{ex}, 0} \sin \gamma_{\mathrm{in}, 0}$ is the non-fluctuating back-action from the SQUID.

From the two-dimensional problem, we can now restrict ourselves to the one-dimensional subspace defined by the preferred tunneling direction [6], which is described by an effective phase $\varphi$. The potential restricted on this direction, $U_{1 \mathrm{D}}(\varphi)$ has the form of a double well $[11,14]$ with stable minima situated at $\pm \varphi_{0}$. In this way, we can expand $U_{1 \mathrm{D}}\left(\varphi, \Phi_{\mathrm{q}}\right) \simeq U\left(\varphi, \Phi_{q}, x\right)+$ $I_{\mathrm{Q}}(\varphi) I_{\mathrm{Q}} M_{\mathrm{SQ}}$. Approximating the phase-dependence of the circulating current as $I_{\mathrm{Q}}(\varphi) \approx I_{\mathrm{p}} \varphi / \varphi_{0}$ where $I_{\mathrm{p}}$ the circulating current in one of the stable 
minima of $\varphi$, we end up with the classical equation of motion of the qubit including the backaction and the friction induced from the SQUID

$$
\begin{aligned}
& {\left[-C_{\mathrm{eff}}\left(\frac{\Phi_{0}}{2 \pi}\right)^{2} \omega^{2}+2 \pi^{2} M_{\mathrm{SQ}}^{2} I_{\mathrm{bias}}^{2} \tan ^{2} \gamma_{\mathrm{in}, 0} \frac{Z_{\mathrm{eff}} I_{\mathrm{p}}^{2}}{i \varphi_{0} \omega \Phi_{0}^{2}}\right] \varphi} \\
& =-\partial_{\varphi} U_{1 \mathrm{D}}\left(\varphi, \Phi_{x, q}+\delta \Phi_{\mathrm{cl}}\right) .
\end{aligned}
$$

From this form, encoded as $D(\omega) \varphi(\omega)=-\partial U / \partial \varphi$ we can use the prescription given in [13] and identify the spectral function for the continuous, classical model as $J_{\text {cont }}=\operatorname{Im} D(\omega)$. From there, we can do the two-state approximation for the particle in a double well [14] and find $J(\omega)$ in analogy to [12]

$$
J(\omega)=\frac{(2 \pi)^{2}}{\hbar \omega}\left(\frac{M_{\mathrm{SQ}} I_{\mathrm{p}}}{\Phi_{0}}\right)^{2} I_{\text {bias }}^{2} \tan ^{2}\left(\frac{\pi \Phi}{\Phi_{0}}\right) \operatorname{Re}\left\{Z_{\mathrm{eff}}(\omega)\right\} .
$$

\subsection{Qubit Dynamics under the Influence of Decoherence}

From $J(\omega)$, we can analyze the dynamics of the system by studying the reduced density matrix, i.e. the density matrix of the full system where the details of the environment have been integrated out, by a number of different methods. The low damping limit, $J(\omega) / \omega \ll 1$ for all frequencies, is most desirable for quantum computation. Thus, the energy-eigenstates of the qubit Hamiltonian, Eq. (1), are the appropriate starting point of our discussion. In this case, the relaxation rate $\Gamma_{r}$ (and relaxation time $\tau_{r}$ ) are determined by the environmental spectral function $J(\omega)$ at the frequency of the level separation $\nu$ of the qubit

$$
\Gamma_{r}=\tau_{r}^{-1}=\frac{1}{2}\left(\frac{\Delta}{\nu}\right)^{2} J\left(\frac{\nu}{\hbar}\right) \operatorname{coth}\left(\frac{\nu}{2 k_{B} T}\right),
$$

where $T$ is the temperature of the bath. The dephasing rate $\Gamma_{\phi}$ (and dephasing time $\tau_{\phi}$ ) is

$$
\Gamma_{\phi}=\tau_{\phi}^{-1}=\frac{\Gamma_{r}}{2}+2 \pi \alpha\left(\frac{\varepsilon}{\nu}\right)^{2} \frac{k_{B} T}{\hbar}
$$

with $\alpha=\lim _{\omega \rightarrow 0} J(\omega) /(2 \pi \omega)$. These expressions have been derived in the context of NMR [15] and recently been confirmed by a full path-integral analysis [10]. In this paper, all rates are calculated for this regime.

For performing efficient measurement, one can afford to go to the strong damping regime. A well-known approach to this problem, the noninteracting blip approximation (NIBA) has been derived in Ref. [13]. This approximation gives good predictions at degeneracy, $\epsilon=0$. At low $|\epsilon|>0$ it contains an artifact predicting incoherent dynamics even at weak damping. At high bias, $\epsilon \gg \Delta$ and at strong damping, it becomes asymptotically correct again. We will not detail this approach here more, as it has been extensively covered in $[11,14]$. 
If $J(\omega)$ is not smooth but contains strong peaks the situation becomes more involved: At some frequencies, $J(\omega)$ may fall in the weak and at others in the strong damping limit. In some cases, whern $J(\omega) \ll \omega$ holds at least for $\omega \leq \Omega$ with some $\Omega \gg \nu / \hbar$, this can be treated approximately: one can first renormalize $\Delta_{\text {eff }}$ through the high-frequency contributions [11] and then perform a weak-damping approximation from the fixed-point Hamiltonian. This is detailed in Ref. [16]. In the general case, more involved methods such as flow equation renormalization [17] have to be used.

\section{Engineering the Measurement Apparatus}

From Eq. 8 we see that engineering the decoherence induced by the measurement apparatus essentially means engineering $Z_{\text {eff }}$. This includes also the contributions due to the measurement apparatus. In this section, we are going to outline and compare several options suggested in literature. We assume a perfect current source that ramps the bias current $I_{\text {bias }}$ through the SQUID. The fact that the current source is non-ideal, and that the wiring to the SQUID chip has an impedance is all modeled by the impedance $Z(\omega)$. The wiring can be engineered such that for a very wide frequency range the impedance $Z(\omega)$ is on the order of the vacuum impedance, and can be modeled by its real part $R_{l}$. It typically has a value of $100 \Omega$.

\section{$3.1 \quad$ R-Shunt}

It has been suggested [18] to overdamp the SQUID by making the shunt circuit a simple resistor $Z(\omega)=R_{\mathrm{S}}$ with $R_{\mathrm{S}} \ll \sqrt{L_{\text {kin }} / 2 C_{\mathrm{J}}}$. This is inspired by an analogous setup for charge qubits, [3]. Following the parameters given in [12], a SQUID with $I_{\mathrm{c}, 0}=200 \mathrm{nA}$ at $\Phi / \Phi_{0} \simeq 0.75$ biased at $I_{\text {bias }}=120 \mathrm{nA}$, we find $L_{\text {kin }} \simeq 2 \cdot 10^{-9} H$. Together with $C_{\mathrm{J}} \simeq 1 \mathrm{fF}$, this means that the SQUID is overdamped if $R \ll R_{\max }=1.4 \mathrm{k} \Omega$. Using Eq. 8, we find that this provides an Ohmic environment with Drude-cutoff, $J(\omega)=\alpha \omega /\left(1+\omega^{2} / \omega_{L R}^{2}\right)$ where $\omega_{L R}=R / L_{\text {kin }}$ and $\alpha=(2 \pi)^{2} / \hbar\left(M_{S Q} I_{q} / \Phi_{0}\right)^{2} I_{\text {bias }}^{2} \tan ^{2}\left(\pi \Phi / \Phi_{0}\right) L_{\text {kin }}^{2} / R_{\mathrm{S}}$. Using the parameters from Ref. [12], $M_{\mathrm{SQ}} I_{q} / \Phi_{0}=0.002$, we find $\alpha R=0.08 \Omega$ and $\omega_{\mathrm{LR}} / R=8.3 \mathrm{GHz} / \Omega$. Thus, for our range of parameters (which essentially correspond to weak coupling between SQUID and qubit), one still has low damping of the qubit from the (internally overdamped) environment at reasonable shunt resistances down to tens of Ohms. For such a setup, one can apply the continuous weak measurement theory as it is outlined e.g. in [18]. This way, one can readily describe the readout through measurement of $Z_{\text {eff }}$ which leaves the system on the superconducting branch. If one desires to read out the state by monitoring the voltage at bias currents above the $I_{\mathrm{c}, \mathrm{eff}}$, our analysis only describes the pre-measurement phase and at least shows that the system is hardly disturbed when the current is ramped. 
a

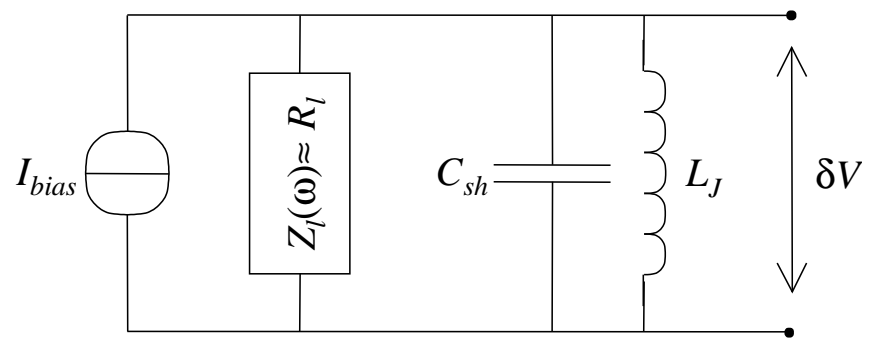

b

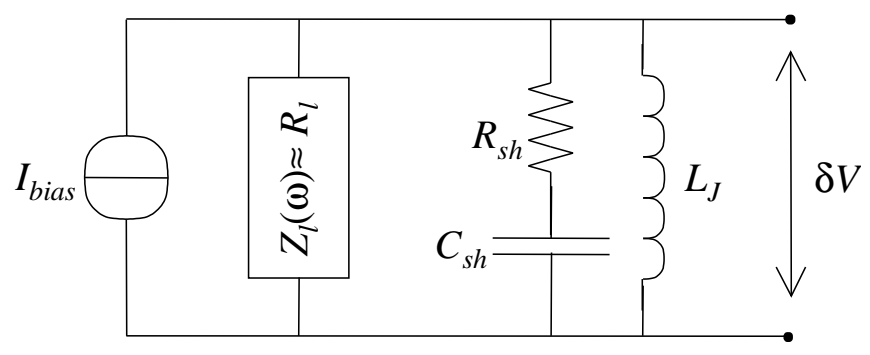

Fig. 2. Circuit models for the $C$-shunted DC-SQUID (a) and the $R C$-shunted DC-SQUID (b). The SQUID is modeled as an inductance $L_{J}$. A shunt circuit, the superconducting capacitor $C_{\mathrm{sh}}$ or the $R_{\mathrm{sh}}-C_{\mathrm{sh}}$ series, is fabricated on chip very close to the SQUID. The noise that couples to the qubit results from JohnsonNyquist voltage noise $\delta V$ from the circuit's total impedance $Z_{\text {eff }}$. $Z_{\text {eff }}$ is formed by a parallel combination of the impedances of the leads $Z_{l}$, the shunt and the SQUID, such that $Z_{\text {eff }}^{-1}=1 / Z_{l}+1 /\left(R_{s h}+1 / i \omega C_{s h}\right)+1 / i \omega L_{J}$, with $R_{s h}=0$ for the circuit (a)

\subsection{Capacitive Shunt}

Next, we consider a large superconducting capacitive shunt (Fig. 2a, as implemented in Refs. [4,9]). The $C$ shunt only makes the effective mass of the SQUID's external phase $\gamma_{\text {ex }}$ very heavy. The total impedance $Z_{\text {eff }}(\omega)$ and $J(\omega)$ are modeled as before, see Fig. 3. As limiting values, we find

$$
\operatorname{Re}\left\{Z_{\mathrm{eff}}(\omega)\right\} \approx\left\{\begin{array}{cl}
\frac{\omega^{2} L_{J}^{2}}{R_{l}}, & \text { for } \omega \ll \omega_{L C} \\
R_{l}, & \text { for } \omega=\omega_{L C} \\
\frac{1}{\omega^{2} C_{s h}^{2} R_{l}}, & \text { for } \omega \gg \omega_{L C}
\end{array}\right.
$$

We can observe that this circuit is a weakly damped $L C$-oscillator and it is clear from (9) and (8) that one should keep its resonance frequency $\omega_{\mathrm{LC}}=1 / \sqrt{L_{\mathrm{J}} C_{\mathrm{sh}}}$, where $\operatorname{Re}\left\{Z_{\text {eff }}(\omega)\right\}$ has a maximum, away from the qubit's 

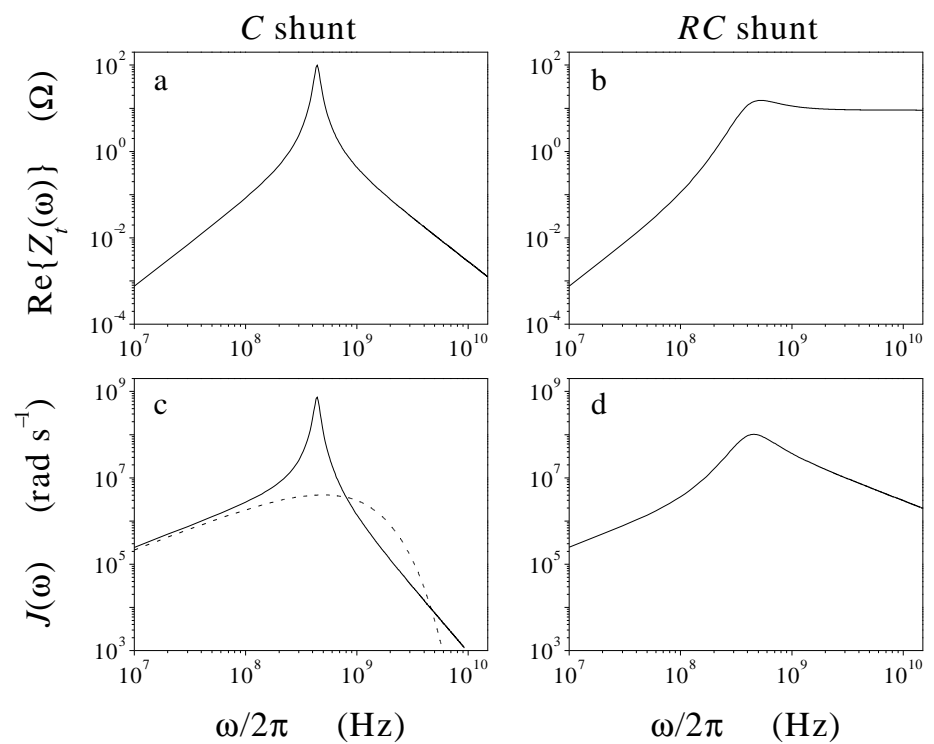

Fig. 3. A typical $\operatorname{Re}\left\{Z_{t}(\omega)\right\}$ for the $C$-shunted SQUID (a) and the $R C$-shunted SQUID (b), and corresponding $J(\omega)$ in (c) and (d) respectively. For comparison, the dashed line in (c) shows a simple Ohmic spectrum, $J(\omega)=\alpha \omega$ with exponential cut off $\omega_{c} / 2 \pi=0.5 \mathrm{GHz}$ and $\alpha=0.00062$. The parameters used here are $I_{\mathrm{p}}=500$ $\mathrm{nA}$ and $T=30 \mathrm{mK}$. The SQUID with $2 I_{c o}=200 \mathrm{nA}$ is operated at $f=0.75 \pi$ and current biased at $120 \mathrm{nA}$, a typical value for switching of the $C$-shunted circuit (the $R C$-shunted circuit switches at higher current values). The mutual inductance $M=8 \mathrm{pH}$ (i. e. $M I_{\mathrm{p}} / \Phi_{0}=0.002$ ). The shunt is $C_{\mathrm{sh}}=30 \mathrm{pF}$ and for the $R C$ shunt $R_{\mathrm{sh}}=10 \Omega$. The leads are modeled by $R_{l}=100 \Omega$

resonance $\omega_{\text {res }}=\nu / \hbar$. This is usually done by chosing $\omega_{\mathrm{LC}} \ll \omega_{\text {res }}$. For a $C$-shunted circuit with $\omega_{L C} \ll \omega_{\text {res }}$, this yields for $J\left(\omega \approx \omega_{\mathrm{LC}}\right)$

$$
J(\omega) \approx \frac{(2 \pi)^{2}}{\hbar \omega^{3}}\left(\frac{M I_{\mathrm{p}}}{\Phi_{0}}\right)^{2} I_{\text {bias }}^{2} \tan ^{2}\left(\frac{\pi \Phi}{\Phi_{0}}\right) \frac{1}{C_{s h}^{2} R_{l}}
$$

The factor $1 / \omega^{3}$ indicates a natural cut-off for $J(\omega)$, which prevents the ultraviolet divergence $[11,10]$ and which in much of the theoretical literature is introduced by hand. Using Eq. 9, we can directly analyze mixing times $\tau_{r}$ vs $\omega_{\text {res }}$ for typical sample parameters (here calculated with the non-approximated version of $\left.\operatorname{Re}\left\{Z_{t}(\omega)\right\}\right)$, see Ref. [12] for details. The mixing rate is then $\Gamma_{r} \approx$ $(2 \pi \Delta / \hbar)^{2} \omega_{r e s}^{-5}\left(M I_{p} / \Phi_{0}\right)^{2} I_{\text {bias }}^{2} \tan ^{2}\left(\pi \Phi / \Phi_{0}\right)\left(2 \hbar C_{s h}^{2} R_{l}\right)^{-1} \operatorname{coth}\left(\hbar \omega_{r e s} / 2 k_{B} T\right)$. With the $C$-shunted circuit it seems possible to get $\tau_{r}$ values that are very long. They are compatible with the ramp times of the SQUID, but too slow for fast repetition rates. For the parameters used here they are in the range of $15 \mu \mathrm{s}$. While this value is close to the desired order of magnitude, one has to be aware of the fact that at these high switching current values the lineariza- 
tion of the junction as a kinetic inductor may underestimate the actual noise. In that regime, phase diffusion between different minima of the washboard potential also becomes relevant and changes the noise properties $[19,20]$.

\subsection{RC-Shunt}

As an alternative we will consider a shunt that is a series combination of a capacitor and a resistor (Fig. $2 \mathrm{~b})$ ( $R C$-shunted SQUID). The $R C$ shunt also adds damping at the plasma frequency of the SQUID, which is needed for realizing a high resolution of the SQUID readout (i. e. for narrow switchingcurrent histograms) [19]. The total impedance $Z_{t}(\omega)$ of the two measurement circuits are modeled as in Fig. 2. For the circuit with the $R C$ shunt

$$
\operatorname{Re}\left\{Z_{t}(\omega)\right\} \approx\left\{\begin{array}{c}
\frac{\omega^{2} L_{J}^{2}}{R_{l}}, \quad \text { for } \omega \ll \omega_{L C} \\
\leq R_{l}, \quad \text { for } \omega=\omega_{L C} \ll \frac{1}{R_{s h C_{s h}}} \\
R_{l} / / R_{s h}, \text { for } \omega=\omega_{L C} \gg \frac{1}{R_{s h} C_{s h}} \\
R_{l} / / R_{s h}, \quad \text { for } \omega \gg \omega_{L C}
\end{array}\right.
$$

The difference mainly concerns frequencies $\omega>\omega_{L C}$, where the $C$-shunted circuit has a stronger cutoff in $\operatorname{Re}\left\{Z_{\text {eff }}(\omega)\right\}$, and thereby a relaxation rate, that is several orders lower than for the $R C$-shunted circuit. Given the values of $J(\omega)$ from Fig. 3 one can directly see from the values of that an $R C$ shunted circuit with otherwise similar parameters yields at $\omega_{\text {res }} / 2 \pi=10$ $\mathrm{GHz}$ relaxation times that are about four orders of magnitude shorter.

\section{Coupled Qubits}

So far, we have applied our modeling only to single qubits. In order to study entanglement in a controlled way and to eventually perform quantum algorithms, this has to be extended to coupled qubits.

\subsection{Hamiltonian}

There is a number of ways how to couple two solid-state qubits in a way which permits universal quantum compuation. If the qubit states are given through real spins, one typically obtains a Heisenberg-type exchange coupling. For other qubits, the three components of the pseudo-spin typically correspond to physically completely distinct variables. In our case, $\hat{\sigma}_{z}$ corresponds to the flux through the loop whereas $\hat{\sigma}_{\mathrm{x} / \mathrm{y}}$ are charges. Consequently, one usually finds Ising-type couplings. The case of $\hat{\sigma}_{\mathrm{y}}^{(1)} \otimes \hat{\sigma}_{\mathrm{y}}^{(2)}$ coupling, i.e. coupling by a component which is orthogonal to all possible single-qubit Hamiltonians, has been extensively studied [21,22], because this type is straightforwardly realized as a tunable coupling of charge qubits [3]. We study the generic case of coupling the "natural" variables of the pseudospin to each other, which 
can be realized in flux qubits using a switchable superconducting transformer [6,23], but has also been experimentally utilized for coupling charge qubits by fixed capacitive interaction [24].

We model the Hamiltonian of a system of two qubits, coupled via Isingtype coupling. Each of the two qubits is described by the Hamiltonian Eq. (1). The coupling between the qubits is described by $\hat{H}_{\mathrm{qq}}=-(K / 2) \hat{\sigma}_{z}^{(1)} \otimes$

$\hat{\sigma}_{z}^{(2)}$ that represents e.g. inductive interaction. Thus, the complete two-qubit Hamiltonian in the absence of a dissipative environment reads

$$
\hat{H}_{2 q b}=-\frac{1}{2} \sum_{i=1,2}\left(\epsilon_{i} \hat{\sigma}_{z}^{(i)}+\Delta_{i} \hat{\sigma}_{x}^{(i)}\right)-\frac{1}{2} K \hat{\sigma}_{z}^{(1)} \hat{\sigma}_{z}^{(2)} .
$$

For two qubits, there are several ways to couple to the environment: Both qubits may couple to a common bath such as picked up by coupling elements [6]. Local readout and control electronics coupling to individual qubits [6] can be described as coupling to two uncorrelated baths. In analogy to the procedure described above, one can determine the spectral functions of these baths by investigating the corresponding impedances.

In the case of two uncorrelated baths, the full Hamiltonian reads

$$
\hat{H}_{2 q b}^{2 b}=\hat{H}_{2 q b}+\sum_{i=1,2} \frac{1}{2} \hat{\sigma}_{z}^{(i)} \widehat{X}^{(i)}+\hat{H}_{B_{1}}+\hat{H}_{B_{2}},
$$

$\widehat{X}^{(i)}=\zeta \sum_{\nu} \lambda_{\nu} x_{\nu}$ are collective coordinates of the bath. In the case of two qubits coupling to one common bath we model our two qubit system in a similar way with the Hamiltonian

$$
\hat{H}_{2 q b}^{1 b}=\hat{H}_{2 q b}+\frac{1}{2}\left(\hat{\sigma}_{z}^{(1)}+\hat{\sigma}_{z}^{(2)}\right) \widehat{X}+\hat{H}_{B}
$$

where $\hat{X}$ is a collective bath coordinate similar to above.

\section{$4.2 \quad$ Rates}

We can derive formulae for relaxation and dephasing rates similar to Eqs. (9) and (10). Our Hilbert space is now four-dimensional. We label the eigenstates as $|E 1\rangle \ldots|E 4\rangle$. We chose $|E 1\rangle$ to be the singlet state $(|\uparrow \downarrow\rangle-|\downarrow \uparrow\rangle) / \sqrt{2}$, which is always an eigenstate [25] whereas $|E 2\rangle \ldots|E 4\rangle$ are the energy eigenstates in the triplet subspace, which are typically not the eigenstates of $\hat{\sigma}_{z}^{(1)}+\hat{\sigma}_{z}^{(2)}$. As we have 4 levels, we have 6 independent possible quantum coherent oscillations, each of which has its own dephasing rate, as well as 4 relaxation channels, one of which has a vanishing rate indicating the existence of a stable thermal equilibrium point. The expressions for the rates, although of similar form as in Eqs. (9) and (10) are rather involved and are shown in [25]. Figure 4 displays the dependence of typical dephasing rates and the sum of all relaxation rates $\Gamma_{R}$ on temperature for the case $\Delta=\epsilon=K=h \nu_{S}$ with 
$\nu_{S}=1 \mathrm{GHz}$. The rates are of the same magnitude for the case of one common bath and two distinct baths. If the temperature is increased above the roll off point set by the intrinsic energy scales, $T_{s}=\left(h / k_{B}\right) \nu_{s}=4.8 \cdot 10^{-2} \mathrm{~K}$, where $E_{s}=1 \mathrm{GHz}$, the increase of the dephasing and relaxation rates follows a linear dependence, indicating that the environmental fluctuations are predominantly thermal. As a notable exception, in the case of one common bath

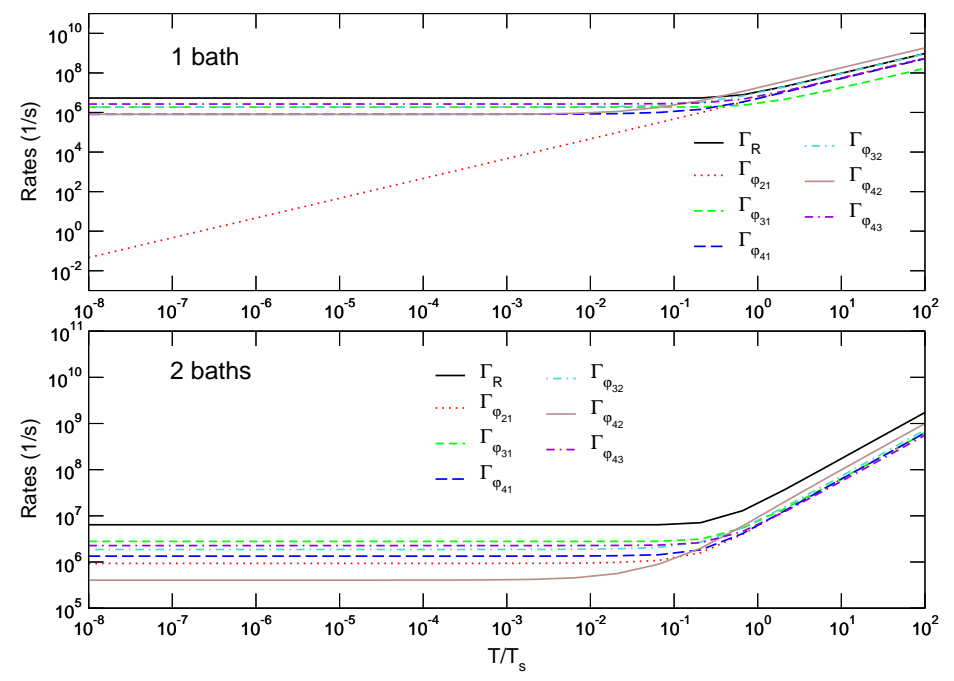

Fig. 4. Log-log plot of the temperature dependence of the sum of the four relaxation rates and selected dephasing rates. Qubit parameters $K, \epsilon$ and $\eta$ are all set to $E_{s}$ and the bath is assumed to be Ohmic $\alpha=10^{-3}$. The upper panel shows the case of one common bath, the lower panel the case of two distinct baths. At the characteristic temperature of approximately $0.1 \cdot T_{s}$ the rates increase very steeply

the dephasing rates $\Gamma_{\varphi_{21}}=\Gamma_{\varphi_{12}}$ go to zero when the temperature is decreased while all other rates saturate for $T \rightarrow 0$. This can be understood as follows: the singlet state $|E 1\rangle$ is left invariant by the Hamiltonian of coupled qubits in a common bath, Eq. (16), i.e. it is an energy eigenstate left unaffected by the environment. Superpositions of the singlet with another eigenstate are usually still unstable, because the other eigenstate generally suffers from decoherence. However, the lowest-energy state of the triplet subspace $|E 2\rangle$ cannot decay by spontaneous emission and flip-less dephasing vanishes at $T=0$, hence the dephasing rate between eigenstates $|E 1\rangle$ and $|E 2\rangle$ vanishes at low temperatures, see Fig. 4. As shown in [25], there can be more "protected" transitions of this kind if the qubit parameters are adjusted such that the symmetry between the unperturbed qubit and the coupling to the bath is even higher, e.g. at the working point for a CPHASE operation. 


\subsection{Gate Performance}

The rates derived in the previous section are numerous and do strongly dependend on the tunable parameters of the qubit. Thus, they do not yet allow a full assesment of the performance as a quantum logic element. A quantitative measure of how well a two-qubit setup performs a quantum logic gate operation are the gate quality factors introduced in [26]: the fidelity, purity, quantum degree and entanglement capability. These factors characterize the density matrices obtained after attempting to perform the gate operation in a hostile environment, starting from all possible initial conditions $\rho(0)=\left|\Psi_{i n}^{j}\right\rangle\left\langle\Psi_{i n}^{j}\right|$. To form all possible initial density matrices needed to calculate the gate quality factors, we use the 16 unentangled product states $\left|\Psi_{i n}^{j}\right\rangle, j=1, \ldots, 16$ defined [22] according to $\left|\Psi_{a}\right\rangle_{1}\left|\Psi_{b}\right\rangle_{2},(a, b=1, \ldots, 4)$, with $\left|\Psi_{1}\right\rangle=|0\rangle,\left|\Psi_{2}\right\rangle=|1\rangle,\left|\Psi_{3}\right\rangle=(1 / \sqrt{2})(|0\rangle+|1\rangle)$, and $\left|\Psi_{4}\right\rangle=(1 / \sqrt{2})(|0\rangle+i|1\rangle)$. They form one possible basis set for the superoperator $\nu_{G}$ which describes the open system dynamics such that $\rho\left(t_{G}\right)=\nu_{G} \rho(0)[22,26]$. The CNOT gate is implemented using rectangular DC pulses and describing dissipation through the Bloch-Redfield equation as described in $[3,25]$.

The fidelity is defined as $\mathcal{F}=(1 / 16) \sum_{j=1}^{16}\left\langle\Psi_{i n}^{j}\left|U_{G}^{+} \rho_{G}^{j} U_{G}\right| \Psi_{i n}^{j}\right\rangle$. The fidelity is a measure of how well a quantum logic operation was performed. Clearly, the fidelity for the ideal quantum gate operation is equal to 1 . The second quantifier is the purity $\mathcal{P}=(1 / 16) \sum_{j=1}^{16} \operatorname{tr}\left[\left(\rho_{G}^{j}\right)^{2}\right]$, which should be 1 in a pure and $1 / 4$ in a fully mixed state. The purity characterizes the effects of decoherence. The quantum degree measures nonlocality. It is defined as the maximum overlap of the resulting density matrix after the quantum gate operation with the maximally entangled Bell-states $\mathcal{Q}=\max _{j, k}\left\langle\Psi_{m e}^{k}\left|\rho_{G}^{j}\right| \Psi_{m e}^{k}\right\rangle$. For an ideal entangling operation, e.g. the CNOT gate, the quantum degree should be 1 . It has been shown [27] that all density operators that have an overlap with a maximally entangled state that is larger than the value 0.78 [22] violate the Clauser-Horne-Shimony-Holt (CHSH) inequality and are thus non-local. The entanglement capability $\mathcal{C}$ is the smallest eigenvalue of the partially transposed density matrix for all possible unentangled input states $\left|\Psi_{i n}^{j}\right\rangle$. (see below). It has been shown [28] to be negative for an entangled state. This quantifier should be -0.5 , e.g. for the ideal CNOT, thus characterizing a maximally entangled final state.

In Fig. 5, the deviations due to decoherence of the gate quality factors from their ideal values are shown. Similar to most of the rates, all gate quality factors saturate at temperatures below a threshold set by the qubit energy scales. The deviations grow linearily at higher temperatures until they reach their theoretical maximum. Comparing the different coupling scenarios, we see that at low temperatures, the purity and fidelity are higher for the case of one common bath, but if temperature is increased above this threshold, fidelity and purity are approximately equal for both the case of one common and two distinct baths. This is related to the fact that in the case of one 

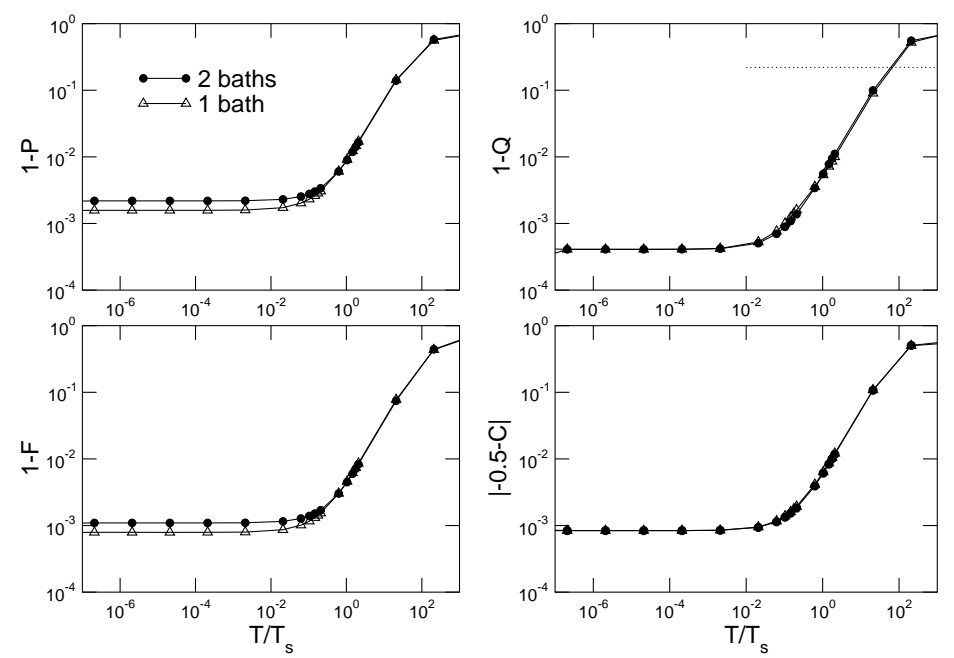

Fig. 5. Log-log plot of the temperature dependence of the deviations of the four gate quantifiers from their ideal values. Here the temperature is varied from $\approx 0$ to $2 \cdot E_{s}$. In all cases $\alpha=\alpha_{1}=\alpha_{2}=10^{-3}$. The dotted line indicates the upper bound set by the Clauser-Horne-Shimony-Holt inequality

common bath all relaxation and dephasing rates vanish during the two-qubitstep of the CNOT (see [25] for details), due to the special symmetries of the Hamiltonian, when temperature goes to zero as discussed above. Still, the quantum degree and the entanglement capability tend towards the same value for both the case of one common and two distinct baths. This is due to the fact that both quantum degree and entanglement capability are, different than fidelity and purity, not defined as mean values but rather characterize the "best" possible case of all given input states.

In the recent work by Thorwart and Hänggi [22], the CNOT gate was investigated for a $\hat{\sigma}_{y}^{(i)} \otimes \hat{\sigma}_{y}^{(j)}$ coupling scheme and one common bath. They find a pronounced degradation of the gate performance with gate quality factors only weakly depending on temperature. If we set the dissipation and the intrinsic energy scale to the same values as in their work, we also observe only a weak decrease of the gate quality factors for both the case of one common bath and two distinct baths in the same temperature range discussed by Thorwart and Hänggi. However, see Fig. 5, overall we find substantially better values. This is due to the fact that for $\hat{\sigma}_{y} \otimes \hat{\sigma}_{y}$ coupling, the Hamiltonian does not commute with the coupling to the bath during the two-qubit steps of the pulse sequence, i.e. the symmetries of the coupling to the bath and the inter-qubit coupling are not compatible. The dotted line in Fig. 5 shows that already at comparedly high temperature, about 20 qubit energies, a quantum degree larger than $\mathcal{Q} \approx 0.78$ can be achieved. Only then, the 
Clauser-Horne-Shimony-Holt inequality is violated and non-local correlations between the qubits occur as described in [22]. Thus, even under rather modest requirements on the experimental setup which seem to be feasible with present day technology, it appears to be possible to demonstrate nonlocality and entanglement between superconducting flux qubits.

\section{Summary}

It has been outlined, how one can model the decoherence of an electromagnetic environment inductively coupled to a superconducting flux qubit. We have exemplified a procedure based on analyzing the classical friction induced by the environment for the specific case of the read-out SQUID. It is shown that the SQUID can be effectively decoupled from the qubit if no bias current is applied. The effect of the decoherence on relaxation and dephasing rates of single qubits has been discussed as well as the gate performance of coupled qubits. We have shown that by carefully engineering the impedance and the symmetry of the coupling, one can reach excellent gate quality which complies with the demands of quantum computation.

We would like to thank M. Governale, T. Robinson, and M. Thorwart for discussions. FKW and MJS acknowledge support from ARO under contractNo. P-43385-PH-QC.

\section{References}

1. see e.g. D. Bouwmeester, A.K. Ekert, and A. Zeilinger, The Physics of Quantum Information (Springer, Berlin, Heidelberg, 2000).

2. D. DiVincenzo, Science 270, 255 (1995).

3. Yu. Makhlin, G. Schön, and A. Shnirman, Rev. Mod. Phys. 73, 357 (2001).

4. I. Chiorescu, Y. Nakamura, C.J.P.M. Harmans, and J.E. Mooij, Science 299, 1869 (2003).

5. Yu.A. Pashkin, T. Yamamoto, O. Astafiev, Y. Nakamura, D.V. Averin, and J.S. Tsai, Nature 421, 823 (2003).

6. J.E. Mooij, T.P. Orlando, L. Levitov, L. Tian, C.H. van der Wal, and S. Lloyd, Science 285, 1036 (1999); T.P. Orlando, J.E. Mooij, L. Tian, C.H. van der Wal, L.S. Levitov, S. Lloyd, and J.J. Mazo, Phys. Rev. B 60, 15398 (1999).

7. L. Tian, L.S. Levitov, C.H. van der Wal, J.E. Mooij, T.P. Orlando, S. Lloyd, C.J.P.M. Harmans, and J.J. Mazo in I. Kulik and R. Elliatiogly, Quantum Mesoscopic Phenomena and Mesoscopic Devices in Microelectronics (Kluwer, Dordrecht, 2000), 429.

8. Yu. Makhlin et al., this volume.

9. C.H. van der Wal, A.C.J. ter Haar, F.K. Wilhelm, R.N. Schouten, C.J.P.M. Harmans, T.P. Orlando, S. Lloyd, and J.E. Mooij, Science 290, 773 (2000).

10. M. Grifoni, E. Paladino, U. Weiss, Eur. Phys. J. B 10, 719 (1999).

11. A.J. Leggett, S. Chakravarty, A.T. Dorsey, M.P.A. Fisher, A. Garg, and W. Zwerger, Rev. Mod. Phys. 59, 1 (1987). 
12. C.H. van der Wal, F.K. Wilhelm, C.J.P.M. Harmans, and J.E. Mooij, Eur. Phys. J. B 31, 111 (2003).

13. A.J. Leggett, Phys. Rev. B 30, 1208 (1984).

14. U. Weiss, Quantum Dissipative Systems, (World Scientific, Singapore, ed. 2, 1999).

15. A. Abragam, Principles of Nuclear Magnetism (Oxford University Press, Oxford, 1961).

16. F.K. Wilhelm, submitted to Phys. Rev. B.

17. S. Kleff, S. Kehrein, and J. von Delft, to appear in Physica E.

18. Yu. Makhlin, G. Schön, and A. Shnirman, Physica C 368, 276 (2002).

19. P. Joyez, D. Vion, M. Götz, M.H. Devoret, D. Esteve, J. Supercond. 12, 757 (1999).

20. W.T. Coffey, Y.P. Kalmykov, J.T. Waldron, The Langevin Equation; with Applications in Chemistry and Electrical Engineering, (World Scientific, Singapore, 1996).

21. M. Governale, M. Grifoni, G. Schön, Chem. Phys. 268, 273 (2001).

22. M. Thorwart, P. Hänggi, Phys. Rev. A 65, 012309 (2002).

23. J.B. Majer, priv. comm. (2002).

24. Yu.A. Pashkin, T. Yamamoto, O. Astafiev, Y. Nakamura, D.V. Averin, J.S. Tsai, Nature 421, 823 (2003).

25. M.J. Storcz and F.K. Wilhelm, Phys. Rev. A 67, 042319 (2003).

26. J.F. Poyatos, J.I. Cirac, P. Zoller, Phys. Rev. Lett. 78, 390 (1997).

27. C.H. Bennett, G. Brassard, S. Popescu, B. Schumacher, J.A. Smolin, and W.K. Wootters, Phys. Rev. Lett. 76, 722 (1996).

28. A. Peres, Phys. Rev. Lett. 77, 1413 (1996). 